\title{
Vortex-Peierls States in Optical Lattices
}

\section{Citation}

Burkov, A. A., and Eugene Demler. 2006. "Vortex-Peierls States in Optical Lattices." Physical Review Letters 96 (18) (May 10). doi:10.1103/physrevlett.96.180406.

\section{Published Version}

doi:10.1103/PhysRevLett.96.180406

\section{Permanent link}

http://nrs.harvard.edu/urn-3:HUL.InstRepos:27896448

\section{Terms of Use}

This article was downloaded from Harvard University's DASH repository, and is made available under the terms and conditions applicable to Other Posted Material, as set forth at http:// nrs.harvard.edu/urn-3:HUL.InstRepos:dash.current.terms-of-use\#LAA

\section{Share Your Story}

The Harvard community has made this article openly available.

Please share how this access benefits you. Submit a story.

\section{Accessibility}




\title{
Vortex-Peierls States in Optical Lattices
}

\author{
A. A. Burkov and Eugene Demler \\ Department of Physics, Harvard University, Cambridge, Massachusetts 02138, USA
}

(Received 14 February 2006; published 10 May 2006)

\begin{abstract}
We show that vortices, induced in cold atom superfluids in optical lattices, may order in a novel vortexPeierls ground state. In such a state vortices do not form a simple lattice but arrange themselves in clusters, within which the vortices are partially delocalized, tunneling between classically degenerate configurations. We demonstrate that this exotic quantum many-body state is selected by an order-from-disorder mechanism for a special combination of the vortex filling and lattice geometry that has a macroscopic number of classically degenerate ground states.
\end{abstract}

DOI: $10.1103 /$ PhysRevLett.96.180406

The existence of quantized vortices is one of the most dramatic manifestations of the macroscopic wave function ("order parameter") in superfluid (SF) Bose gases. Considerable theoretical [1] and experimental [2] effort has thus been applied to the study of SF vortices in cold atoms systems. Vortices are usually regarded as classical objects that form regular lattices due to long-range repulsive interactions between them. Intuitively, this point of view seems to be in accord with the fact that vortices are topological objects, i.e., the existence of an isolated vortex in the system can be established by observing phase winding infinitely far away from the vortex core. However, analogies with two-dimensional (2D) electronic systems in high magnetic fields, exhibiting the fractional quantum Hall $(\mathrm{FQH})$ effect, suggest that under certain conditions vortex lattices may be melted by quantum fluctuations, and various strongly correlated vortex liquid states may thus emerge [3].

Optical lattices offer additional opportunities to explore the quantum mechanical behavior of vortices by allowing one to tune the strength of quantum fluctuations. Several approaches to stabilizing FHQ states in cold atoms systems using optical lattices have already been proposed [4-6]. More generally, of interest are situations in which vortices may behave as strongly interacting quantum particles, moving in a periodic optical lattice potential. Such a possibility appears to be rather counterintuitive, since naïvely we think of vortices as macroscopic objects. Nevertheless, the common view nowadays is that vortices in 2D systems can be considered as quantum particles with a finite mass [7]. It is then interesting to find experimentally observable phenomena in cold atoms systems in which this quantum nature of vortices is manifested. In particular, manifestations of the quantum mechanical behavior of vortices may be most dramatic in situations when long-range intervortex interactions are frustrated, which strongly enhances the effect of quantum fluctuations. Such a frustration in optical lattice systems may be engineered by choosing the appropriate combination of the optical lattice geometry and the vortex density. We should point out that some classical commensuration effects between vortex lattices and the underlying optical lattice pinning potential have already
PACS numbers: 05.30.Jp, 03.75.Lm, 67.90.+z, 74.81.Fa

been studied, both theoretically [8] and experimentally [9], but the possible quantum effects have not been previously considered.

In this Letter we discuss an example of such a quantum mechanical behavior of SF vortices in an optical lattice. We show that for a particular combination of the optical lattice geometry and the vortex filling, for which classical vortex configurations are strongly frustrated, a vortexPeierls (VP) state is realized. By VP state we mean a vortex lattice, in which vortices are not localized at the maxima of the optical lattice potential, but are instead partially delocalized, resonating quantum mechanically between degenerate pinned configurations. Peierls ordering has been extensively studied, most recently in the context of quantum magnetism of localized spin systems [see Ref. [10] for review]. Here we demonstrate that such ordering may, under certain conditions, occur for vortices in optical lattice superfluids.

We consider a SF system of cold bosonic atoms, loaded in an optical periodic potential with the dice lattice geometry, shown in Fig. 1. The fascinating features of the quantum mechanics of particles on the dice lattice in a

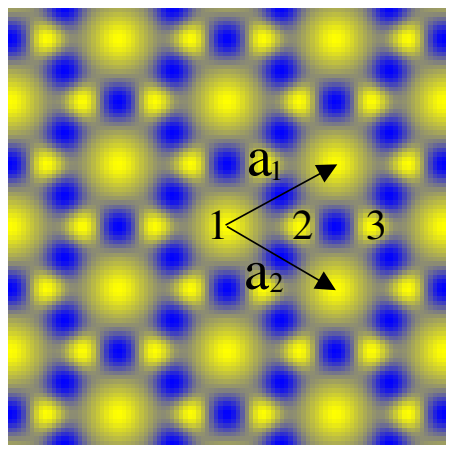

FIG. 1 (color online). Optical dice lattice created by superimposing three laser field potentials $I_{1}(\mathbf{r}), I_{2}(\mathbf{r})$, and $I_{3}(\mathbf{r})$ (see text). $\mathbf{a}_{1}=\frac{3}{2} \hat{x}-\frac{\sqrt{3}}{2} \hat{y}$ and $\mathbf{a}_{2}=\frac{3}{2} \hat{x}+\frac{\sqrt{3}}{2} \hat{y}$ are the basis directions. Lighter areas correspond to potential minima. Inequivalent sites in the three-site unit cell of the dice lattice are labeled as 1, 2 , and 3 . 
perpendicular magnetic field were first pointed out by Vidal et al. [11] and extensively studied in a number of subsequent works [see, e.g., [12] and references therein]. Atoms in an optical lattice can be described by the BoseHubbard model [13], in which bosons are assumed to tunnel between nearest-neighbor sites of the lattice and interact when they are on the same site. We will assume that the average number of bosonic atoms per site of the dice lattice $\bar{n}$ is an integer. In this case increasing the onsite interaction energy $U$ relative to the hopping amplitude $t$ until $U / \bar{n} t \sim 1$ will induce a SF-Mott Insulator (MI) transition [14] when $\bar{n}$ bosons will be localized on each site to minimize the interaction energy.

To induce vortices in the SF we add an effective perpendicular "magnetic field." We will comment on particular methods that could be used to create such an effective field at the end of the Letter. We will focus on a specific value of the flux per plaquette of the dice lattice $2 \pi f=2 \pi / 3$, where $f=1 / 3$ has the physical meaning of the vortex filling, i.e., the average number of vortices per plaquette. Centers of dice plaquettes can be associated with sites of the dual kagomé lattice, which correspond to the maxima of the optical lattice potential. It is then convenient to assume that the vortex cores are located on the kagomé lattice sites. Since vortices interact via a long-range repulsive potential, they will try to arrange themselves in patterns on the kagomé lattice that maximize the distance between each vortex and its neighbors. As shown by Korshunov [15], at filling factor $f=1 / 3$ the set of vortex configurations that minimize the classical interaction energy between the vortices consists of all states, where every triangular plaquette of the kagomé lattice is occupied by exactly one vortex. The number of such configurations grows exponentially with the system size and the classical ground state of vortices has only algebraic order at zero temperature, but no true long-range order [15].

Nevertheless, below we will demonstrate that strong quantum fluctuations near the SF-MI transition lift the classical degeneracy and select a vortex state with a true long-range order. This state has, however, a manifestly quantum mechanical nature, in that the vortices are not simply localized on sites of the kagomé lattice, but are instead partially delocalized over plaquettes, thus exhibiting what we call a VP ordering.

To approach the problem analytically, we will take advantage of the presumed proximity of our system to the SF-MI critical point. Since the bosons are at an integer filling, the standard Landau-Ginzburg (LG) theory can be applied not too far away from the critical point. The imaginary time LG functional of the SF order parameter fields is given by [16]:

$$
\begin{aligned}
S= & \int_{0}^{\beta} d \tau\left[-J \sum_{i \mu}\left(\Phi_{i}^{*} \Phi_{i+\mu} e^{-i A_{i \mu}}+\text { c.c. }\right)\right. \\
& \left.+\sum_{i}\left(\left|\partial_{\tau} \Phi_{i}\right|^{2}+r\left|\Phi_{i}\right|^{2}+u_{4}\left|\Phi_{i}\right|^{4}+u_{6}\left|\Phi_{i}\right|^{6}\right)\right]
\end{aligned}
$$

Here $\Phi_{i}$ is the local SF order parameter, $\mu$ denote the nearest-neighbor vectors of the dice lattice, $J \sim U \bar{n} t$, $u_{4}, u_{6}>0$, and $r$ tunes the system across the SF-MI transition. To find the ordering patterns near the transition, we need to diagonalize the first term in Eq. (1) [17]. Choosing Landau gauge for the vector potential $A_{i \mu}=2 \pi f i_{x}(1-$ $\delta_{\mu x}$ ) one obtains the following dispersion for the lowest Hofstadter band: $\boldsymbol{\epsilon}(\mathbf{k})=-J\left[6+\cos \left(k_{1}\right)+\cos \left(k_{2}\right)-\right.$ $\left.2 \cos \left(k_{1}-k_{2}\right)+\sqrt{3} \sin \left(k_{1}\right)+\sqrt{3} \sin \left(k_{2}\right)\right]^{1 / 2}$, where $\mathbf{k}=$ $k_{1} \mathbf{b}_{1}+k_{2} \mathbf{b}_{2}$ and $\mathbf{b}_{1,2}=\frac{1}{3} \hat{x} \pm \frac{1}{\sqrt{3}} \hat{y}$ are the reciprocal lattice vectors of the dice lattice. The boson dispersion has two minima inside the first Brillouin zone of the dice lattice, at wave vectors $\mathbf{k}_{0}=(0,2 \pi / 3)$ and $\mathbf{k}_{1}=(2 \pi / 3,0)$. The corresponding eigenvectors are given by $v^{0}=(1,1,0)$ and $v^{1}=\left(e^{-2 \pi i / 3}, 0,1\right)$. We can then write the lattice order parameter fields $\Phi_{i}$ as linear combinations of these two low energy modes $\Phi_{\sigma}\left(\mathbf{r}_{i}\right)=\sum_{\ell=0,1} \varphi_{\ell} v_{\sigma}^{\ell} e^{i \mathbf{k}_{\ell} \cdot \mathbf{r}_{i}}$, where the index $i$ labels the unit cells of the dice lattice, $\sigma=1,2,3$ labels sites within each unit cell, and $\varphi_{\ell}$ are the fields, corresponding to the low energy boson modes. To obtain the LG action in terms of the fields $\varphi_{\ell}$ in its most general form we need to know how these fields transform under the symmetry operations of the dice lattice. The relevant operations are the elementary translations along the basis directions $T_{1}$ and $T_{2}$, rotations by $\pi / 3$ around the sixfold coordinated sites $R_{\pi / 3}$, and reflections with respect to the $x$ and $y$ axes $I_{x, y}$. These transformations are given by: $T_{1}: \varphi_{\ell} \rightarrow \varphi_{\ell} e^{-2 \pi i \ell / 3}, T_{2}: \varphi_{\ell} \rightarrow \varphi_{\ell} e^{2 \pi i(\ell-1) / 3}$, $R_{\pi / 3}: \varphi_{\ell} \rightarrow \varphi_{\ell+1} e^{-2 \pi i(\ell+1) / 3}, \quad I_{x}: \varphi_{\ell} \rightarrow \varphi_{\ell}^{*} e^{-2 \pi i \ell / 3}$, $I_{y}: \varphi_{\ell} \rightarrow \varphi_{\ell+1}^{*} e^{2 \pi i / 3}$, where the subscripts of the fields are taken modulo 2. Using these transformations, the most general form of the imaginary time LG action is found to be [18]

$$
\begin{aligned}
S= & \int_{0}^{\beta} d \tau \int d \mathbf{r}\left\{\sum_{\ell}\left[\left|\partial_{\tau} \varphi_{\ell}\right|^{2}+c^{2}\left|\partial_{\mu} \varphi_{\ell}\right|^{2}+r\left|\varphi_{\ell}\right|^{2}\right]\right. \\
& +u_{4}\left(\sum_{\ell}\left|\varphi_{\ell}\right|^{2}\right)^{2}+v\left|\varphi_{0}\right|^{2}\left|\varphi_{1}\right|^{2} \\
& \left.+u_{6}\left(\sum_{\ell}\left|\varphi_{\ell}\right|^{2}\right)^{3}+w\left[\left(\varphi_{0}^{*} \varphi_{1}\right)^{3}+\text { c.c. }\right]\right\} .
\end{aligned}
$$

In the mean-field approximation, which we expect to be accurate for the effective classical $2+1$-dimensional system, described by Eq. (2), three different SF phases are possible, depending on the signs of the $v$ and $w$ couplings.

(1) $v>0$ : either $\left|\varphi_{0}\right| \neq 0$ or $\left|\varphi_{1}\right| \neq 0$. (2) $v<0, w<0$ : $\left|\varphi_{0}\right|=\left|\varphi_{1}\right| \neq 0$. The relative phase $\theta=\arg \left[\varphi_{0}^{*} \varphi_{1}\right]$ is determined by the last term in Eq. (2) and is given by $\theta=$ $2 \pi n / 3, n=0,1,2$. (3) $v<0, w>0:\left|\varphi_{0}\right|=\left|\varphi_{1}\right| \neq 0$. The relative phase is given by $\theta=(2 n+1) \pi / 3, n=$ $0,1,2$. To reinterpret the states we have found in the vortex language, it is convenient to calculate gauge-invariant supercurrents on each bond, which we define as $J_{i \mu} \sim$ $\operatorname{Im}\left(\Phi_{i}^{*} \Phi_{i+\mu} e^{-i A_{i \mu}}\right)$. In the $v>0$ state we find that supercurrents vanish on every bond. This fact, combined with 
the picture of this state in terms of the SF order parameter, leads to the vortex configuration shown in the right panel of Fig. 2. Since this state contains configurations, in which two vortices are located on nearest-neighbor sites, it cannot be the true ground state of the vortices.

Calculating supercurrents in the second candidate ground state, realized when $v, w<0$, we obtain the configuration shown in the right panel of Fig. 3. Vortices in this case are localized on the dice lattice plaquettes, which have supercurrents circulating around them in the counterclockwise direction. This configuration is a member of the classical ground state manifold, since none of the vortices have nearest neighbors.

Finally, the supercurrent pattern in the state, realized when $v<0$ and $w>0$, is shown in the right panel of Fig. 4. One can see that none of the dice plaquettes in this case have a full vortex localized in it. Instead, the vortices appear to be bound in partially delocalized triplets, populating the six dice lattice plaquettes, adjacent to the sixfold coordinated sites with zero order parameter expectation values. The fact that the order parameter vanishes on these sites means that vortices are moving around such sites, locally destroying phase coherence. It also means that the boson number does not fluctuate on such sites, i.e., the bosons on these sites are in the Mott phase. The only vortex state that is consistent with this picture, and also does not violate the constraint of no-nearest-neighbor vortices, is the one in which the vortex triplets resonate between two degenerate configurations (corresponding instantaneous order parameter phase configurations are explicitly shown in Fig. 4). This state is clearly more energetically favorable than the state in Fig. 3, since it does not violate the no-nearest-neighbor constraint but also allows the vortices to gain kinetic energy by partially

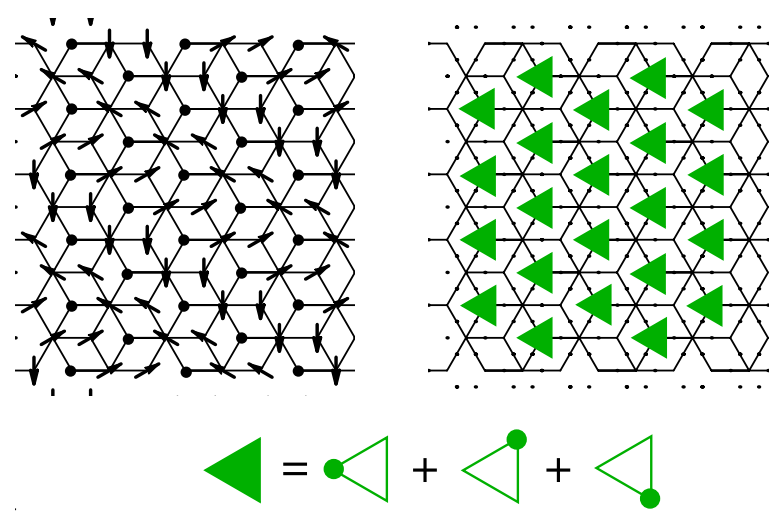

FIG. 2 (color online). (Left) Order parameter configuration corresponding to the $v>0$ state. Direction of each arrow represents the phase of the corresponding order parameter field, while length represents the magnitude. Expectation values of the order parameter vanish on all sites of type 3 (shown by dots). (Right) Corresponding vortex configuration. Shaded triangles contain a vortex, uniformly delocalized over the three sites of each triangle. Note that the supercurrents vanish on every bond of the dice lattice. delocalizing over hexagonal plaquettes of the dual kagomé lattice. We can estimate the energy gain in this state due to the vortex delocalization, compared to the $w<0$ state, as follows. Interaction energy of the vortices (energy of the supercurrents) is of order $\bar{n} t$ per lattice site. Vortex kinetic energy (energy gain from phase fluctuations) is of order $U$ per site. Therefore, the energy gain in the $w>0$ state, compared to the $w<0$ state, is of order $\bar{n} t(U / \bar{n} t)^{3}$, which is not small when $U \sim \bar{n} t$. Thus, we find that the state in Fig. 4 is the ground state of the vortices in our problem. Since the vortices are not localized on sites of the kagomé lattice, but are partially delocalized over plaquettes, we call this state a VP state.

Let us now discuss how to observe the VP state experimentally. Creating an optical lattice with a dice geometry experimentally is more difficult than most other 2D lattices, but fortunately still possible with the current technology. We propose the following procedure [see Ref. [12] for an alternative proposal]. One first creates a kagomé lattice, which is done using the laser field potential proposed in Ref. [19]: $\quad I_{1}(\mathbf{r})=\sum_{i=1}^{3}\left[\cos \left(\mathbf{k}_{i} \cdot \mathbf{r}+\frac{\pi \sigma_{i}}{2}\right)+2 \cos \left(\frac{1}{3} \mathbf{k}_{i}\right.\right.$. $\left.\left.\mathbf{r}+\frac{3 \pi \sigma_{i}}{2}\right)\right]^{2}, \quad$ where $\quad \mathbf{k}_{1}=(\pi, \sqrt{3} \pi), \quad \mathbf{k}_{2}=(\pi,-\sqrt{3} \pi)$, $\mathbf{k}_{3}=(-2 \pi, 0), \sigma_{1}=\sigma_{3}=1$, and $\sigma_{2}=-1$. Here the maxima of the potential correspond to the kagomé lattice sites. To create a perfect dice lattice we superimpose two additional laser potentials that have a triangular lattice geometry: $\quad I_{2}(\mathbf{r})=4 \sum_{i=1}^{3} \cos ^{2}\left(\frac{1}{3} \mathbf{k}_{i} \cdot \mathbf{r}\right), \quad$ and $\quad I_{3}(\mathbf{r})=$ $-4 \sum_{i=1}^{3} \cos ^{2}\left(\mathbf{g}_{i} \cdot \mathbf{r}\right), \quad$ where $\quad \mathbf{g}_{1}=(\pi, \pi / \sqrt{3}), \quad \mathbf{g}_{2}=$ $(0,-2 \pi / \sqrt{3})$, and $\mathbf{g}_{3}=(-\pi, \pi / \sqrt{3})$. Here $I_{2}(\mathbf{r})$ has maxima at the sixfold coordinated sites of the dice lattice, while $I_{3}(\mathbf{r})$ has minima at both threefold and sixfold coordinated sites. The superposition of $I_{1}(\mathbf{r}), I_{2}(\mathbf{r})$, and $I_{3}(\mathbf{r})$ creates a perfect dice lattice, in which all potential wells have equal depth. Effective magnetic flux in this setup can be created by the rotating mask method [9], which generates a rotating optical lattice potential.

Alternatively, the proposal of Ref. [4] can be used to create the effective perpendicular field. One uses the combination of a time-dependent quadrupolar potential $V(t)=$ $V_{\mathrm{qp}} \sin (\omega t) x y$ and a temporal modulation of the tunneling
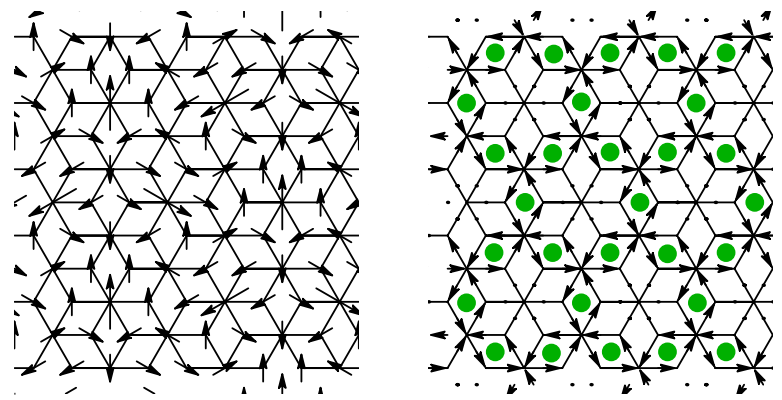

FIG. 3 (color online). (Left) Order parameter configuration corresponding to the $v, w<0$ state. (Right) Corresponding supercurrent configuration. Plaquettes, containing vortices (shown by circles), are the ones that have supercurrents circulating around them in the counterclockwise direction. 

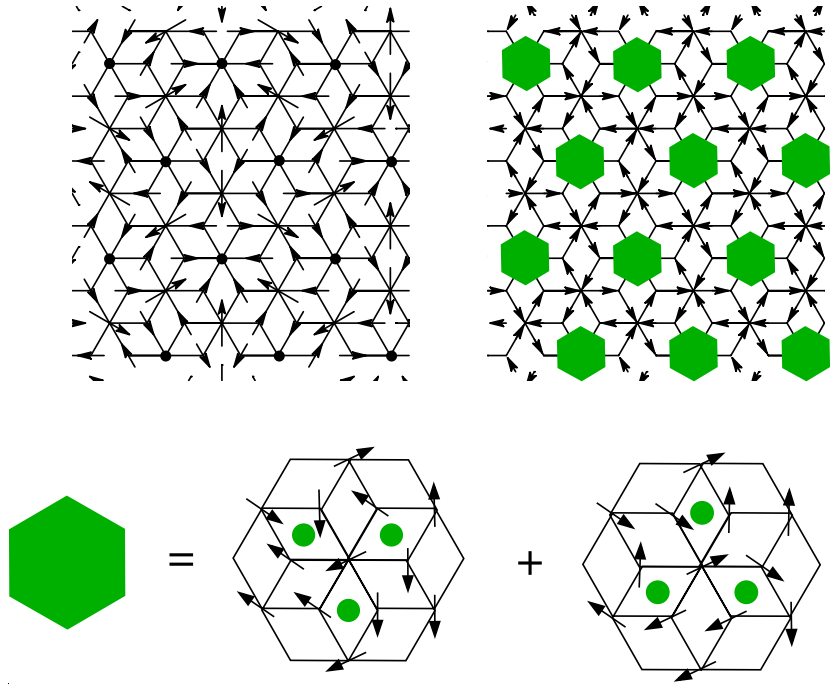

FIG. 4 (color online). (Left) Order parameter configuration corresponding to the $v<0, w>0$ state. Expectation value of the order parameter vanishes on some of the sixfold coordinated sites (shown by dots). (Right) Corresponding supercurrent configuration. Note that none of the plaquettes contain a full vortex. Instead, vortices are bound into triplets populating the groups of six plaquettes, adjacent to sixfold coordinated sites with zero order parameter expectation value. Below, SF order parameter phase snapshots, corresponding to the two resonating vortex triplet configurations are shown.

amplitudes for different nearest-neighbor directions in the dice lattice. Modulation of the tunneling amplitudes can be achieved in our case by varying the strengths of the three components of the $I_{3}(\mathbf{r})$ potential. To achieve the flux of $2 \pi / 3$ per plaquette, the parameters of the quadrupolar potential have to be chosen such that $V_{\mathrm{qp}} / \hbar \omega=\pi / 2 \sqrt{3}$. In this setup, time-of-flight interference imaging can be used to study the periodic structure of the vortex lattice. Lattice periodicity, however, is not enough to distinguish the VP state we found from the state in Fig. 3, since they have identical reciprocal lattice vectors. VP ordering can be detected by analyzing nontrivial noise correlations [20] that will be present in the time-of-flight image. Namely, in addition to the sharp peaks in the density distribution (i.e., first order correlation function), corresponding to the reciprocal lattice vectors $\mathbf{g}_{i} / 3$ of the vortex lattice, one should observe strong incoherent background. Such a background would be absent in a regular superfluid, in which all vortices are strictly localized. This is due to the fact that the vortex motion locally destroys phase coherence on some sites of the optical lattice. In the case of the VP state, described above, the incoherent background contains correlations at the same wave vectors $\mathbf{g}_{i} / 3$, which may be found by measuring the density autocorrelation function. If the perpendicular field is created by the rotating mask technique, one can use interference between two identical corotating condensates [21] to image the vortex lattice configurations.
In conclusion, we have proposed that vortices in optical lattice SF may exist in VP ground states, which are direct analogs of valence-bond-solid states of interacting bosons. In particular, we have demonstrated that in the case of a dice optical lattice with vortex filling of $1 / 3$ per plaquette, the ground state of the vortices is a plaquette VP state, in which vortices bind into triplets that resonate between two degenerate configurations on plaquettes of the dual kagomé lattice. Such unconventional vortex ordering is a result of an order-by-disorder phenomenon, where extensive degeneracy of frustrated classical vortex configurations is lifted by quantum fluctuations.

We would like to thank E. Altman, M. Greiner, B. I. Halperin, M. D. Lukin, R. G. Melko, and V. Schweikhard for useful discussions. Financial support was provided by the National Science Foundation under Grants No. DMR02-33773 and No. DMR01-32874.

[1] A. L. Fetter and A. A. Svidzinsky, J. Phys. Condens. Matter 13, R135 (2001), and references therein.

[2] J. Abo-Shaeer, C. Raman, J. M. Vogels, and W. Ketterle, Science 292, 476 (2001); I. Coddington, P. Engels, V. Schweikhard, and E. A. Cornell, Phys. Rev. Lett. 91, 100402 (2003).

[3] N. K. Wilkin and J. M. F. Gunn, Phys. Rev. Lett. 84, 6 (2000); T.-L. Ho, ibid. 87, 060403 (2001); B. Paredes et al., ibid. 87, 010402 (2001); J. Sinova et al., ibid. 89, 030403 (2002).

[4] A.S. Sørensen et al., Phys. Rev. Lett. 94, 086803 (2005).

[5] E. J. Mueller, Phys. Rev. A 70, 041603 (2004).

[6] D. Jaksch and P. Zoller, New J. Phys. 5, 56 (2003).

[7] See, e.g., R. Fazio and H. van der Zant, Phys. Rep. 355, 235 (2001), and references therein.

[8] C. Wu et al., Phys. Rev. A 69, 043609 (2004); J. W. Reijnders and R. A. Duine, Phys. Rev. Lett. 93, 060401 (2004); H. Pu et al., ibid. 94, 190401 (2005); R. Bhat et al., Phys. Rev. Lett. 96, 060405 (2006).

[9] V. Schweikhard et al. (to be published).

[10] S. Sachdev, in Quantum Magnetism, edited by U. Schollwock et al., Lecture Notes in Physics (Springer, New York, 2004).

[11] J. Vidal et al., Phys. Rev. Lett. 81, 5888 (1998).

[12] M. Rizzi et al., cond-mat/0510341 (unpublished).

[13] D. Jaksch et al., Phys. Rev. Lett. 81, 3108 (1998).

[14] M. Greiner et al., Nature (London) 415, 39 (2002).

[15] S. E. Korshunov, Phys. Rev. Lett. 94, 087001 (2005).

[16] E. Altman and A. Auerbach, Phys. Rev. Lett. 89, 250404 (2002).

[17] D. Blankschtein et al., Phys. Rev. B 30, 1362 (1984).

[18] K. Sengupta et al., cond-mat/0601175 [Phys. Rev. B (to be published)].

[19] L. Santos et al., Phys. Rev. Lett. 93, 030601 (2004).

[20] E. Altman et al., Phys. Rev. A 70, 013603 (2004).

[21] A. Polkovnikov et al., Proc. Natl. Acad. Sci. U.S.A. 103, 6125 (2006). 